\title{
A Study on the Quadcopter Position Control using Low-Cost MEMS Imperference Sensor
}

\author{
Min-Seok Jie ${ }^{1}$, Seung-Hun Kim² and Won-Hyuck Choi ${ }^{3 *}$ \\ ${ }_{1,3}$ Department of Avionics Engineering Hanseo University \\ 236-49, Gomseom-ro, Nam-myeon, Taean-gun, Chungcheongnam-do, \\ Republic of Korea 32158 \\ ${ }^{2}$ Department of Aeronautical Systems Engineering Hanseo University \\ 236-49, Gomseom-ro, Nam-myeon, Taean-gun, Chungcheongnam-do, \\ Republic of Korea 32158 \\ 1,3 \{choiwh, jiems\}@hanseo.ac.kr, ${ }^{2}$ kimsh753@naver.com
}

\begin{abstract}
In this paper, a low-cost MEMS (Micro Electro Mechanical Systems) inertial sensor is used to obtain the attitude information of the quadcopter. In the process of calculating the tilt angle of the sensor against the zero-point, bias error, it generates an error and causes disturbance of movement. To reduce the noise and error, we used the complementary filter to collect the attitude information of the quadcopter and to reduce the noise and the error to calculate the accurate attitude information.
\end{abstract}

Keywords: MEMS, Quadcopter, Low cost, Sensor, Attitude control

\section{Introduction}

On unmanned aircraft, there are several sections in the category Multi-Copter, such as Tri-copter, Quart Copter, and Hex-copter Octa-copter, and at least two propellers are required to stabilize the vehicle. These gases are not limited in time or space when they are landed vertically. However, it takes a lot of force to reach a certain altitude, and a lot of time to get to a stable landing.

Drones, which are vertical takeoffs and landings of the UCSCS: unmanned Aerial Vehicle systems, are used for a variety of purposes and make it easy for dangerous or difficult for humans to gain access.

Quadro Rotor, a compound word used in this study, is a Quadro Rotor type heater that weighs less than other unmanned aerial vehicles, making it a versatile choice. It is receiving much attention in many industrial areas, such as investigating or monitoring disasters that are inaccessible to humans, or studying and transporting environmental fields.

Recently, quadcopter is the most popular application for aerial photography. As the technology has developed, small size sensors and cameras have been released, which makes it much easier to take unmanned aerial photographs because the constraints on weight are reduced. It is also used for agricultural purposes, and can be taken off and landing with only the farm area setting. As a hobby, many DJs in China have produced many quad-copters that can be manipulated through programs [3].

The advantage of such a quad-copter is that it is free and easy to maintain as well as landing. Therefore, the missions that the existing helicopter was assigned to are becoming increasingly quad-copter. However, quad-copter is also influenced by air currents or external factors.

The greater the amount of movement due to external factors, the more battery is consumed and the more difficult it is to control. In addition, due to the zero-point, bias error,

Received (November 14, 2017), Review Result (March 20, 2018), Accepted (March 26, 2018) 
and drift phenomenon, many cumulative errors are generated in the process of calculating the inclination angle of the sensor, resulting in disturbance of motion. In order to perform hovering, the quad-copter must sense the disturbance caused by the disturbance and maintain the correct attitude and position. In order to overcome this disadvantage, the attitude control is important [4].

The quad-copter controls the relative position of the four rotors to control the attitude and movement of the gas. By using Quadcopter's MEMS inertial sensor, posture control can basically be based on gyro and acceleration data.

In this paper, we investigate attitude control of quad - copter by using low - cost MEMS (Micro Electro Mechanical Systems) inertial sensor to collect attitude information of quad - copter and reduce noise and error. In Chapter 2, we describe the components and the driving principle of the quad-copter. Section 3 describes the filter implementation and simulation and describes the results of the actual experiment. Section 4 presents the conclusion and future work.

\section{Components and Driving Principle}

Quadcopter components are divided into several categories depending on their purpose, and devices are being developed to match unmanned aircraft.

This chapter describes the most basic building blocks and operating principles required for flight for this study.

\subsection{Components}

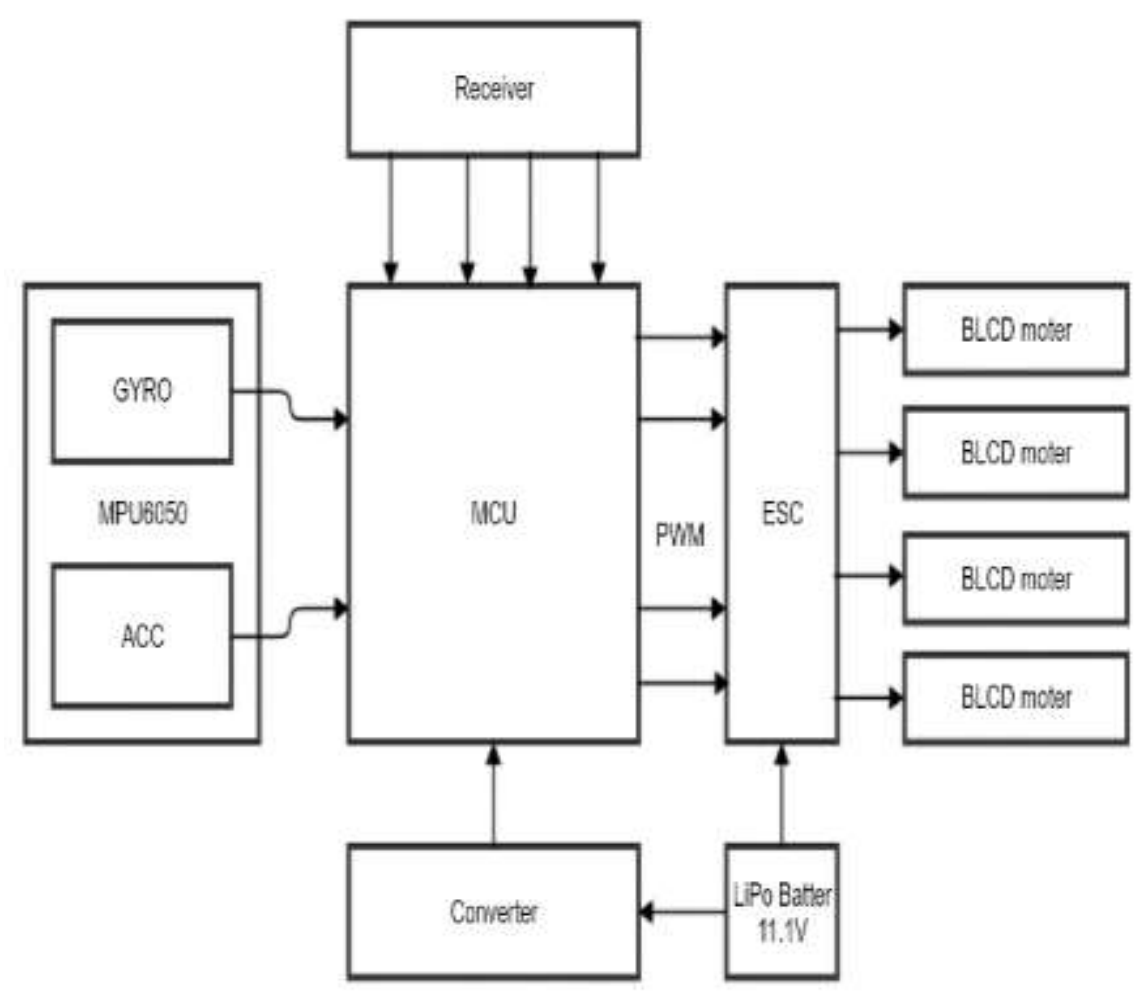

Figure 1. The Basic Components of the Quad-Copter

The basic components of the Quadcopter are: It is composed of MCU (Micro Controller Unit), Gas (Frame), Battery, Receiver (ECS), Transmission (Electronic Speed Controls), and Brushless DC electric motor (BLDC: Brushless Direct Current). 
(1) Quadcopter Frame

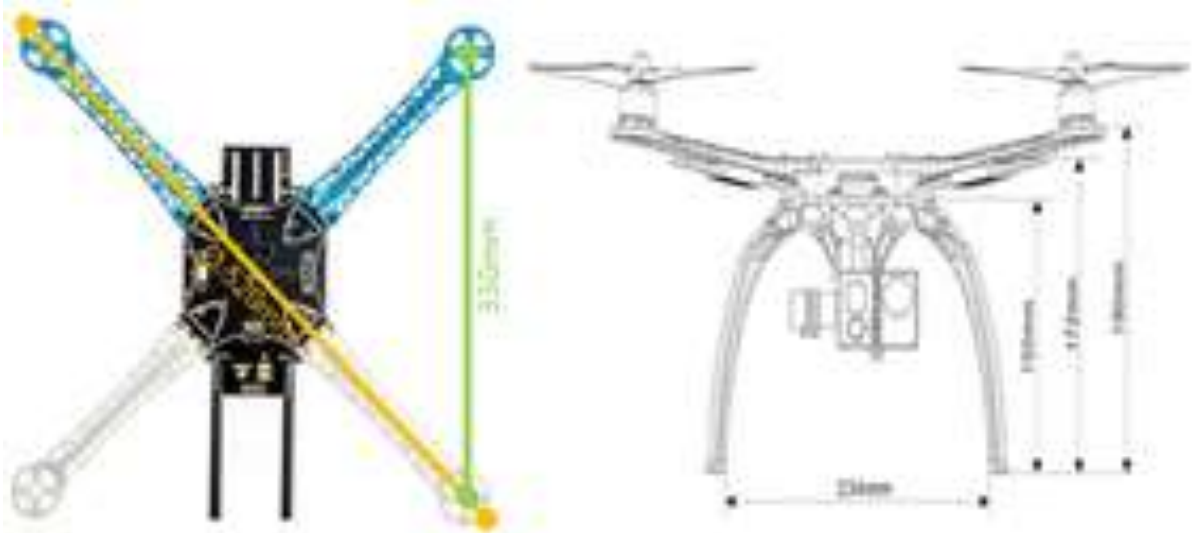

Figure 2. Frame Specification

In quadcopters, a frame is one of the things that can make a difference in weight. This is because the weight of the gas varies with the type of material in the frame and the design method. The heavier the gas is, the more stable it is when flying, but when it is over a certain weight, the slower it is moving and the faster it is used by the battery.

The frame used in this study was the FPV S50-material made of plastic and composite materials, making it an asymmetric structure that makes the weight of the gas lighter. The airframe is $520 \mathrm{~mm}$ in diameter as shown in Figure 2, $330 \mathrm{~mm}$ in distance between the front and rear motors, and $170 \mathrm{~mm}$ in distance from the top frame to the ground surface.

(2) MCU (Micro Controller Unit)

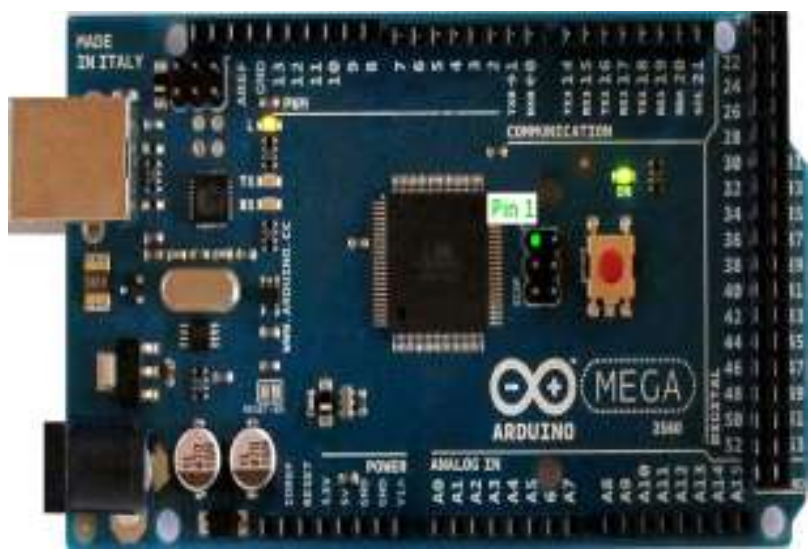

Figure 3. ATmega 2560 (Arduino MEGA)

One of the MCU types used in this study was the Arduino Mega with AT Mega 2560. The AT Mega 2560 is the Atmel Corporation's 8-bit microcontroller and is used in many fields. The AT Mega 2560 specification includes a 32 x 8bit Universal Register and an Peripheral Control Register, and it has ISO Flash Memory Collector to enable programs internally. The ISP also enables firmware to be available in both the application and boot domains. Peripheral devices feature four 8-bit PWM (pulse width modulated) channels and two to 16bit PWM channels, each capable of being programmed with 12 programs. These functions process information, formulas, and filters from sensors that come from quadcopters. 
(3) IMU (Inertial Measurement Unit)

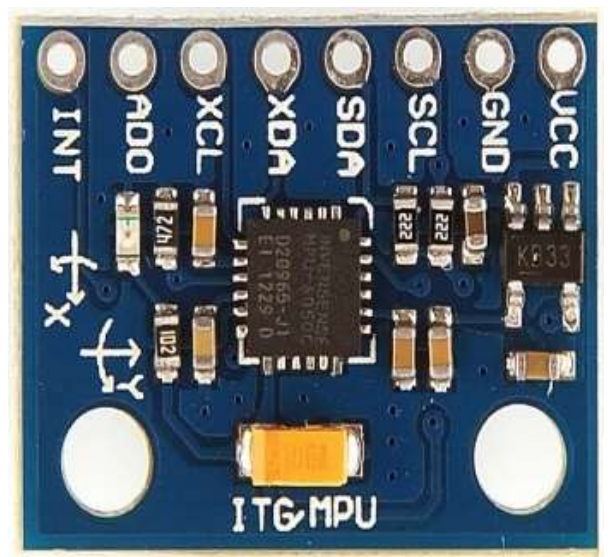

Figure 4. MPU-6050 (GY-521)

The low-value MEMS inertia sensor used in this paper is a silicon chip that is specialized by integrating in mechanical parts as well as a micro-electron control technique (MEMS). Sensors that pass through this process are inexpensive and small [5].

MPU 6050 is a six-down (DOF) sensor that contains both acceleration and gyro sensors in one sensor. Data can be extracted using the I2C (Inter-Integration Circuit) communication protocol. It is used to measure and convey the position information of quadcopters. Data are transferred to MCU.

\section{(4) Actuator}

The actuators are the most important parts of the Quadcopter, and they are motors and propellers and transmissions (ECS), and so on.

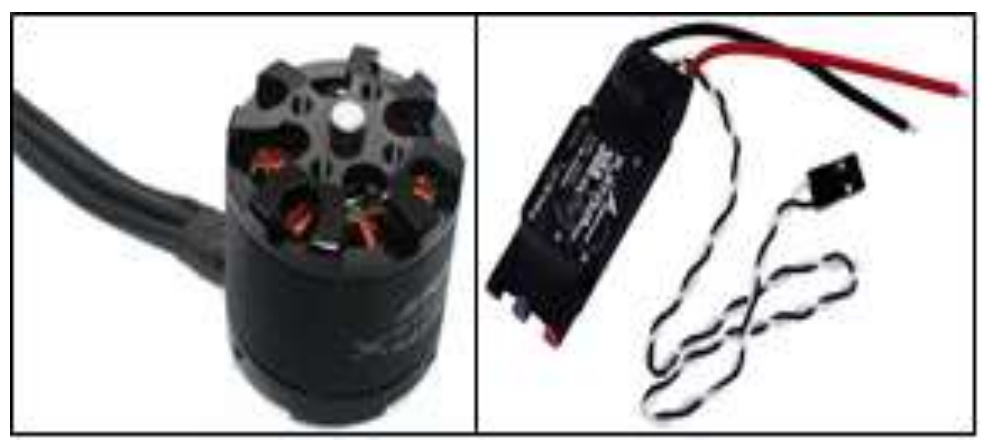

Figure 5. BLDC Motor(Left), ESC(Right)

The motor uses a brushless motor rather than a conventional DC motor, and the BLDC motor consists of a permanent magnet on top of it and consists of a rotor and a winding. Electric lines are used to convert electrical energy into mechanical energy by emitting electrical currents, turning the rotor by magnetic fields.

In addition, the control of the motor speed in BLDC motor is controlled by the Electronic Speed Controller (ESC) and is referred to as a transmission. The Electronic Speed Controller (ESC) uses the product shown in the figure to the right of Figure 5. The Electronic Speed Controller (ESC) uses a three-phase frequency applied by the power from the battery to produce a three-phase frequency so that the brushless motor can use a threephase converter. 


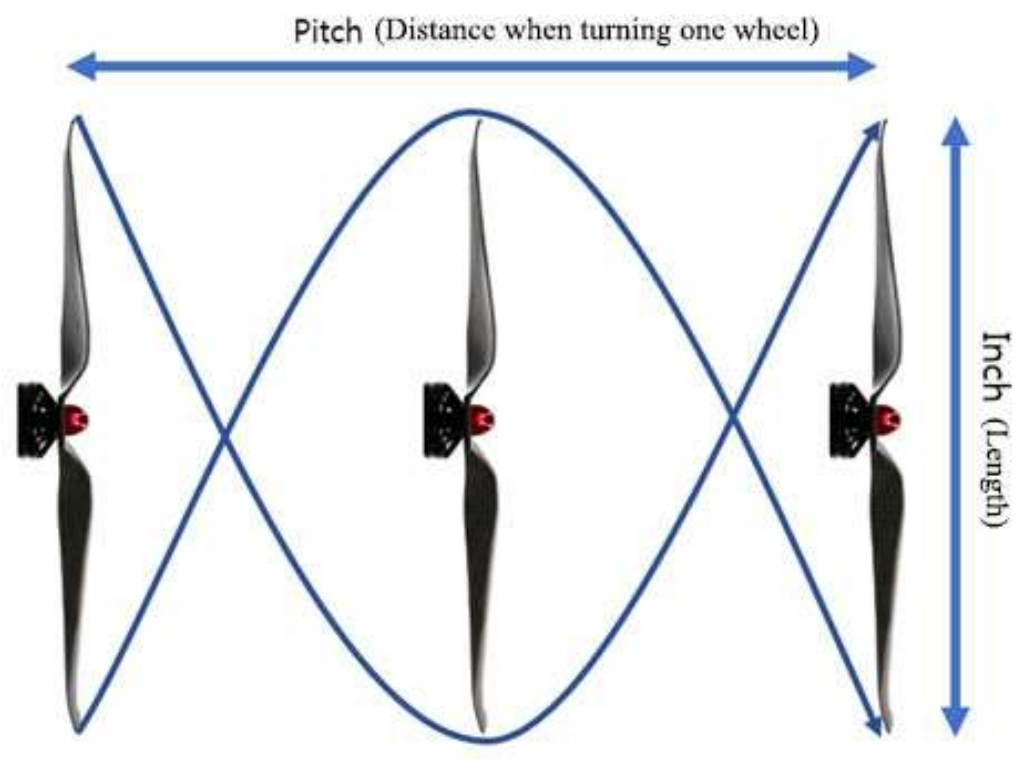

Figure 6. Propeller Diameter and Pitch

Propeller is basically expressed in four-letter figures. The two front seats represent the diameter of the propeller, while the two rear seats are called the pitch, which is the length in which the propeller moves forward during a turn. Figure 6 shows the propeller (s) used in this study, 1024, with a 2.4-inch pitch value in 10 inches.

\subsection{Principle of Operation}

The quadcopter's principle of operation is not affected by the number of turns in the propeller. There are three types of PITCH, ROLL, and YAW for flight purposes, and the axes are divided into $\mathrm{X}, \mathrm{Y}$, and $\mathrm{Z}$. When the four motors have the same rotational speed, they can be raised or lowered when the same thrust is produced, and they can be lifted and landed vertically.
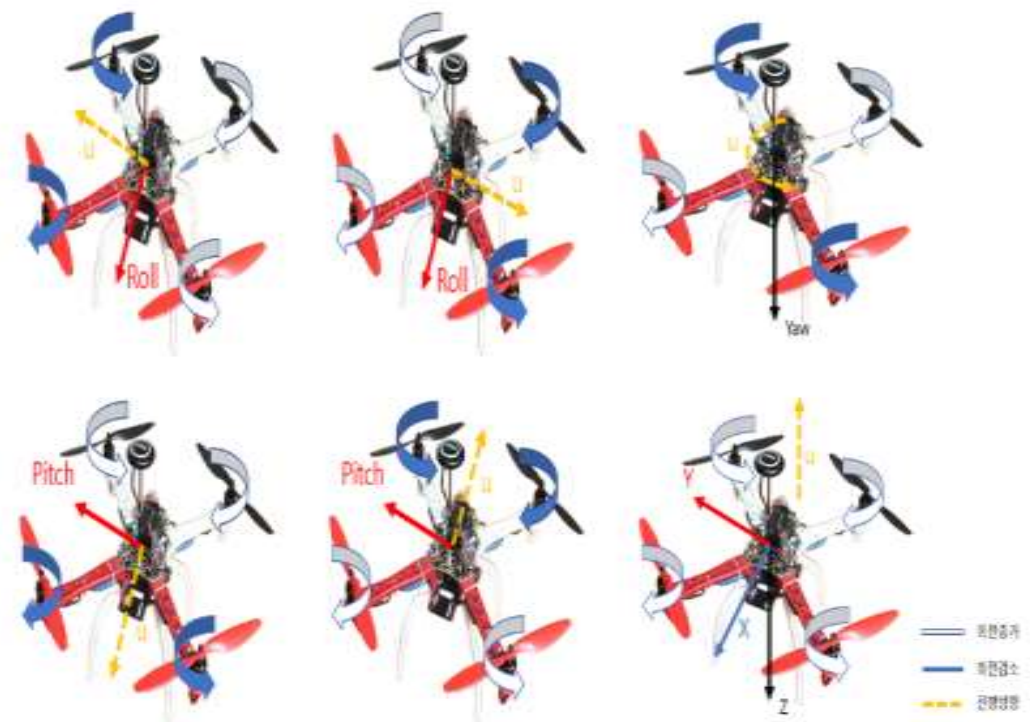

Figure 7. Quadcopter Operating Principle 


\section{Dynamic and Position Control Experiment}

\subsection{Coordinates and Dynamics of Quadrotors}
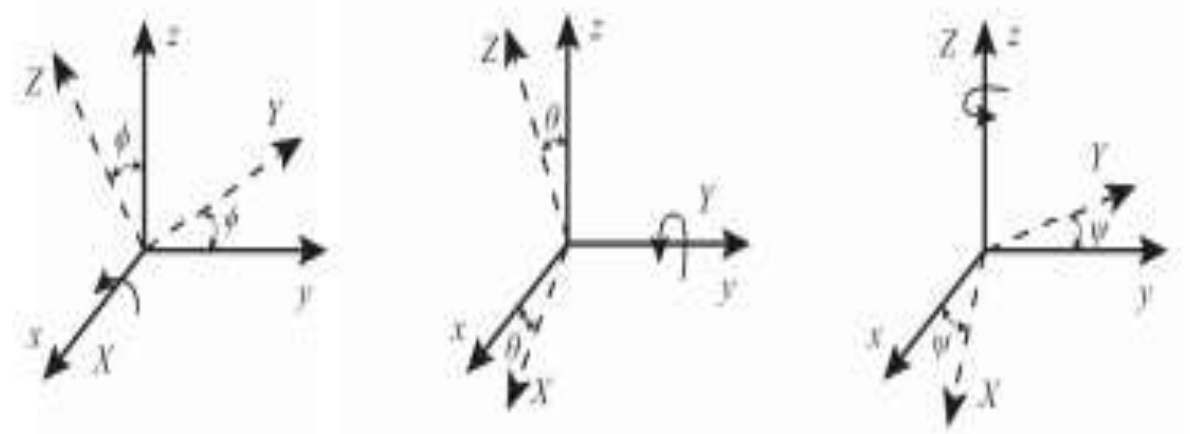

Figure 8. Rotation Coordinates for Fixed Coordinate System

$R_{z y x}=R_{z}(\psi) R_{y}(\theta) R_{x}(\phi)$
$\cos \theta \cos \psi$
$\left[\begin{array}{ccc}\cos \phi \sin \psi & -\sin \theta \\ \sin \phi \sin \theta \cos \psi-\cos \phi \sin \psi & \sin \phi \sin \theta \sin \psi+\cos \phi \cos \psi & \sin \phi \cos \theta \\ \cos \phi \sin \theta \cos \psi+\sin \phi \sin \psi & \cos \phi \sin \theta \sin \psi-\sin \phi \cos \psi & \cos \phi \cos \theta\end{array}\right]$

The quadcopter's dynamic modeling can be expressed as shown in Figure 8, and can also be represented in the inertia coordinate system as shown in the expression below (2). In the expression (2), it denotes the position, the angle of illumination, the velocity and the angular velocity.

$\varphi_{\mu \nu}(z)=\frac{\left\|k_{\mu \nu}\right\|^{2}}{\sigma^{2}} e^{-\frac{\left\|k_{\mu \nu}\right\|^{2}\|z\|^{2}}{\sigma^{2}}}\left[e^{i k_{\mu \nu} z}-e^{\frac{-\sigma^{2}}{2}}\right]$

The position of the strainer and the angle of Euler can be expressed with the velocity and acceleration values shown in the Gas Coordinate System (3). In the expression (3), R represents the matrix for turning the gas coordinate system against the inertia coordinate system, and $\mathrm{C}$ is the matrix that describes the relationship between the inertia coordinate system and the velocity component of the Euler valve and the angular velocity vector of the gas coordinate system. If the equation (3) is distinguished, the following expression (4) is used:

$$
\begin{aligned}
& \varphi_{\mu v}(z)=\frac{\left\|k_{\mu \nu}\right\|^{2}}{\sigma^{2}} e^{-\frac{\left\|k_{\mu \nu}\right\|^{2}\|z\|^{2}}{\sigma^{2}}}\left[e^{i k_{\mu \nu} z}-e^{\frac{-\sigma^{2}}{2}}\right] \\
& \varphi_{\mu \nu}(z)=\frac{\left\|k_{\mu \nu}\right\|^{2}}{\sigma^{2}} e^{-\frac{\left\|k_{\mu \nu}\right\|^{2}\|z\|^{2}}{\sigma^{2}}}\left[e^{i k_{\mu v} z}-e^{\frac{-\sigma^{2}}{2}}\right]
\end{aligned}
$$

If the distance of the motor from its center of gravity is equal to, and the torque coefficient for thrust is equal, then the thrust produced from the Quadcopter's actuator is the same as the total thrust applied on the gas stationary coordinate system [5]. 


\subsection{Posture Control Experiment}

(1) MEMS inertial sensor

The inertia sensor is made up of no more than a noise or a thermal vibration, so a collision with an air molecule vibration the sensor to produce a noise. These phenomena create zero variations and are caused by accumulated error by calculation [9].

As mentioned above, the data measured in the gyro sensor cannot be used as it causes drift due to cumulative error caused by calculation when integrating to obtain an angle.

The acceleration sensor has a large variation in the error range, making the position information less precise. They are also sensitive to shock, vibration and external forces. It is difficult to extract practical slope information, and it is not possible to measure gradient values by means of acceleration sensors alone under an unstopped motion.

These sensors take advantage of the advantages and disadvantages of two sensors. However, even if the sensor is fused, it is likely to use filters due to continuous drift phenomena and errors [5] [9].

\section{(2) Complementary filter}

As described above, the complementary filter causes a drift phenomenon of the gyro sensor, and an acceleration sensor also exhibits an error. For this reason, the advantages of the gyro sensor and the acceleration sensor are complemented to compensate for the disadvantages and a fusion method using a complementary filter (High Pass Filter + Low Pass Filter) [5].

$\varphi_{\mu \nu}(z)=\frac{\left\|k_{\mu \nu}\right\|^{2}}{\sigma^{2}} e^{-\frac{\left\|k_{\mu \nu}\right\|^{2}\|z\|^{2}}{\sigma^{2}}}\left[e^{i k_{\mu \nu} z}-e^{\frac{-\sigma^{2}}{2}}\right]$

In (5), the value obtained by removing the error bias of the gyro sensor value is (PHI_dot), and the angle measured from the acceleration sensor is expressed by (PHI_a). The equation (5) can be expressed by the following equation (6).

The obtained equation (6) is shown in Figure 9 below.

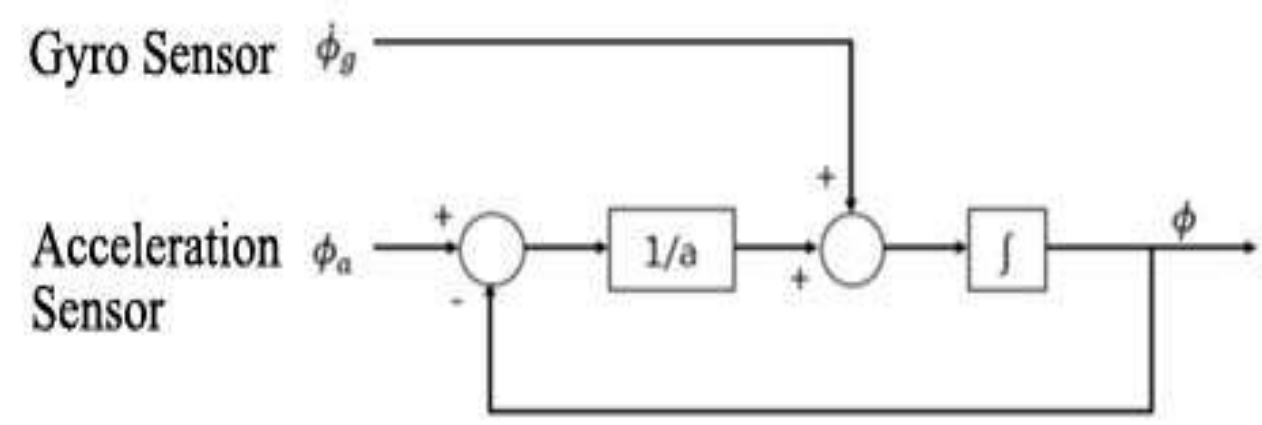

Figure 9. Complementary Filter Block Diagram

In the block diagram, the angle (PHI_dot) measured by the gyro sensor is directly integrated into (PHI), and the angle measured by the acceleration sensor (PHI_a) is multiplied by $1 /$ a to integrate it into (PHI). It should be noted that the gyro sensor value is the angular velocity value based on the coordinate system, and the acceleration value is the angular value based on the global coordinate system. 


\section{(3) Filter simulation and experiment}

The simulation was run before experimenting with the complementary filter on an actual quad-copter. The complementary filter is one of the most used filters for sensor fusion. It is simple and easy to design, and unlike the Kalman filter, there is no fear of divergence. However, the disadvantage is that the frequency characteristics of the sensors to be fused are complementary to each other.

In order to simulate the filter, the gyro sensor passes through HPF (High Pass Filter) and the acceleration sensor is added after LPF (Low Pass Filter) filter. Figure 10 below shows the result of simulating the complementary filter in MATLAB.

The figure below shows the drift phenomenon in which the value of the gyro sensor continues to drop. This drift phenomenon can be confirmed by the fact that the drift value disappears while passing through the complementary filter.

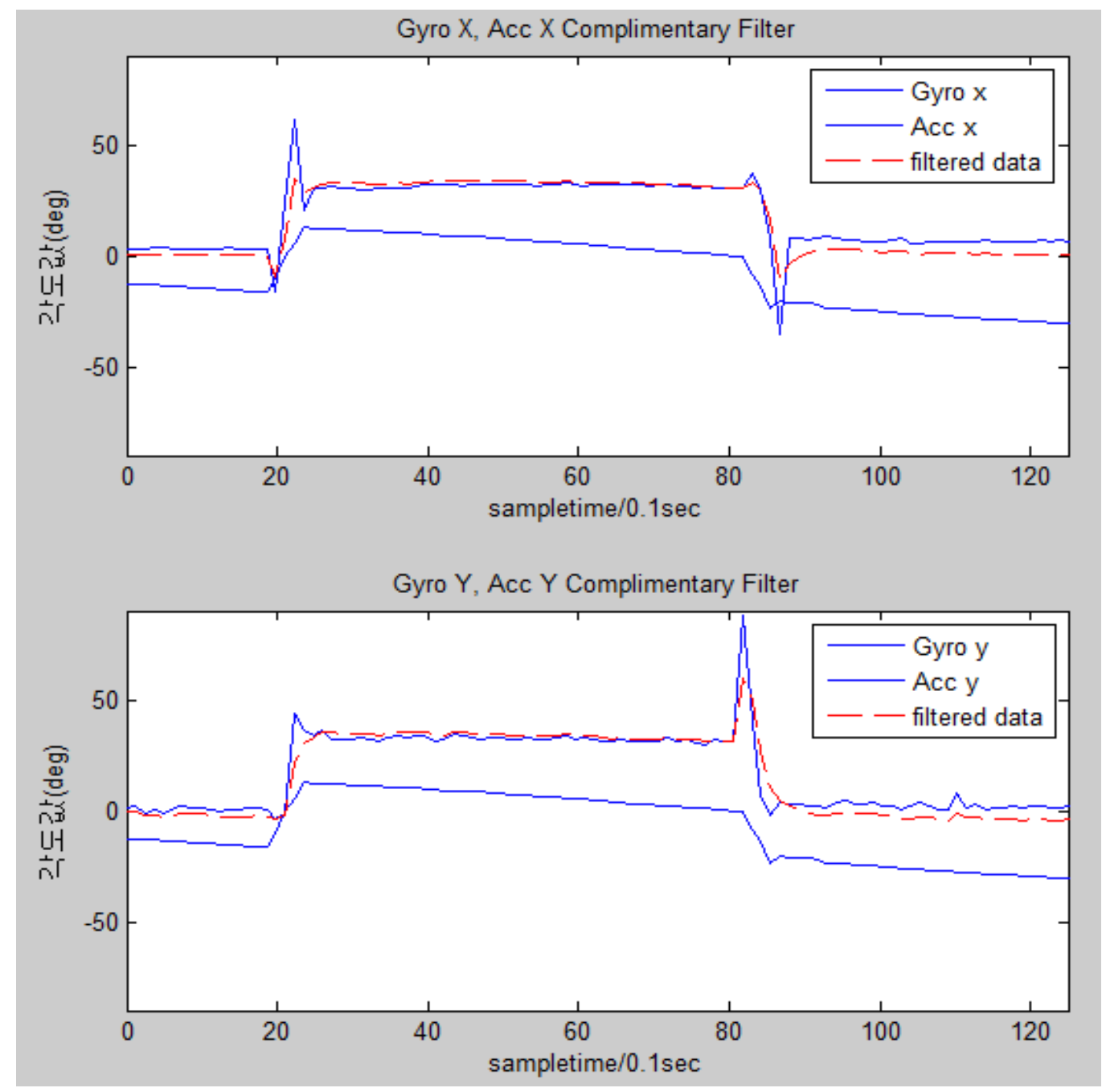

Figure 10. Complement Filter Simulation

Based on the simulated results, we experimented on the quad - copter. The graphs shown below were obtained by directly applying a complementary filter on the ATmega2560 board on the Quad Copter. In the graph shown in Figure 9 below, the filter is applied when the vibration is severe at the value where the divergence changes, and the divergence and vibration do not occur, and the resultant value is reached. 


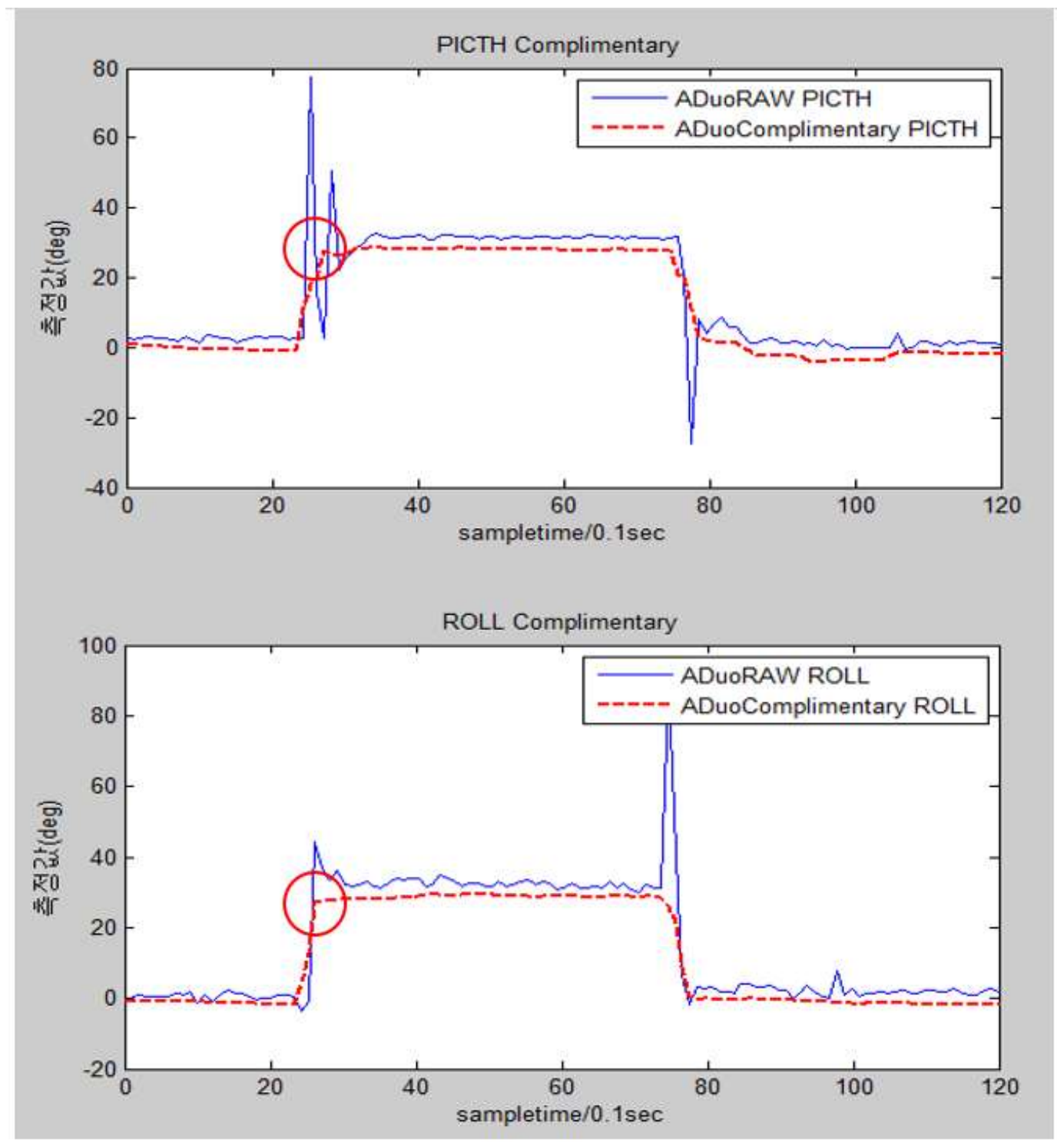

Figure 11. Quad Copter (Arduino) Complementary Filter

\section{Conclusion}

In quad-copter, attitude control is affected by external factors, which means that the more the battery is consumed, the more difficult it is to control. In addition, due to the zero-point, bias error, and drift phenomenon, many cumulative errors are generated in the process of calculating the inclination angle of the sensor, resulting in disturbance of motion.

We found that the attitude information of the quadcopter with a lot of vibration and noise was not accurate and the value changed every second. Values that include noise or disturbance can interfere with controlling posture and can lead to confusion by transmitting erroneous information. A complementary filter was applied to eliminate this cumulative error. As a result of applying the filter, it was confirmed that vibration and noise were eliminated.

In future work, we will study the effect of the complementary filter and the Kalman filter on the posture control by comparing the two results and applying the two filters together.

In addition, this study will be helpful for the posture control of the quadcopter in the future. 


\section{References}

[1] S. R. Lee, H. Berry, O. Temam and M. Lipasti, "Performance improvement of WDM channels using inline dispersion management in transmission links with OPC placed at various position", The Journal of Korea Navigation Institute, vol. 14, no. 5, (2100), pp. 668-676.

[2] H. Kim, H. S. Jeong, K. T. Chong and D. J. Lee, "Dynamic Modeling and Control Techniques for MultiRotor Flying Robots", Transactions of the Korean Society of mechanical engineers, vol. 38, no. 2, (2014), pp. 137-148.

[3] G. Y. Lee, "The drones come quickly to us", The Korean Institute of Electrical Engineers, vol. 65. no. 1, (2016), pp. 17-24.

[4] H. W. Lim, M. S. Jie and W. H. Choi, "Filter Study for SFAC Development, Quad-copter Safety Improvement System", International Journal of Control and Automation, vol. 140, (2017), pp. 124-128.

[5] H. G. Min, "Design of Complementary Filter using MEMS-type Gyroscope and Accelerometer", Ph.D. Changwon Univ, 20 Changwondaehak-ro Uichang-gu Changwon-si Gyeongsangnam-do Republic of Korea, (2011).

[6] Y. H. Jo, "Enhanced Quadcopter Modeling based Attitude and Velocity Estimation Filter", M.D, Sejong Univ, 209, Neungdong-ro, Gwangjin-gu, Seoul, Republic of Korea, (2016).

[7] J. H. Lee, "Implementation on the Attitude-controller of Quad-rotor Using ATmega128, M.D. Hoseo Unvi, 20 Hoseo-ro 79beon-gil Baebang-eup Asan-si Chungnam, Republic of Korea, (2015).

[8] A. Hashmi, H. Berry, O. Temam and M. Lipasti, "Multi-copter Modeling and Simulation", The Korean Institute of Industrial Engineers, Korea: KOR, (2016), pp. 1-10.

[9] D. H. Yu, D. Y. Lim, N. O. Sel, J. H. Park and K. T. Chong, "Study of Sensor Fusion for Attitude Control of a Quad-rotor", Institute of Control, Robotics and Systems, vol. 21, no. 5, (2015), pp. 453-458.

\section{Authors}

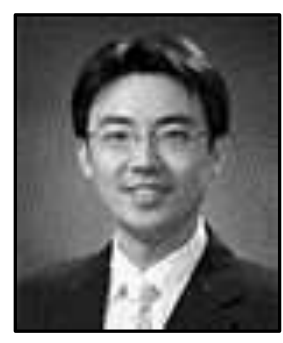

Min-Seok Jie, he graduated from the Ph.d degree in the department of avionics Engineering from Korea Aerospace University in 2006. he is Hanseo University Avionics Engineering Professor in to the present in 2008 .

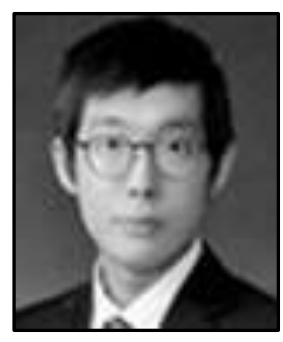

Seung-Hun Kim, he is a master's degree in Aeronautical System Engineering, Hanseo University. He graduated from the Department of Aeronautical and Mechanical Engineering, Hanseo University. He is studying Kalman filters, embedded systems, and smart network communications.

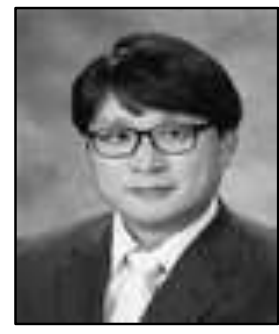

Won-Hyuck Choi (Corresponding author), he received the Ph.D. degree in avionics from Korea Aerospace University, Korea. He was a Professor of smart network at Doowon Technical University College, Korea. currently, he is Professor of avionics at Hanseo University, Korea, where he has been since 2014. His present research interests include embedded system, home network. 